\title{
Analytical Model and Data-driven Approach for Concrete Moisture Prediction
}

\author{
Karthick Thiyagarajan and Sarath Kodagoda \\ Centre for Autonomous Systems, University of Technology Sydney, Australia. \\ E-mail: Karthick.Thiyagarajan@student.uts.edu.au, $\underline{\text { Sarath.Kodagoda@uts.edu.au }}$
}

\begin{abstract}
-
The advent of smart sensing technologies has opened up new avenues for addressing the billion dollar problem in the wastewater industry of $\mathrm{H}_{2} \mathrm{~S}$ corrosion in concrete sewer pipes, where there is a growing interest in monitoring the environmental properties that govern the rate of corrosion. In this context, this paper proposes a methodology to predict the moisture content of concretes through data-driven approach by using Gaussian Process Regression modeling. The experimental program in this study practices measurements during wetting and drying phases of concrete. The obtained moisture data is used to train the prediction model against interpreted electrical resistivity data. The data of analytical model formulated from Archie's Law is then analyzed with experimental and Gaussian Process prediction data.
\end{abstract}

Keywords -

Analytical Model; Data-driven approach; Drying-wetting process; Electrical resistivity data; Gaussian Process prediction model; Moisture data.

\section{Introduction}

Sewer concrete corrosion is now widely recognized as a significant global problem by water utilities. This problem is vastly influenced by the gas concentration of Hydrogen Sulphide $\left(\mathrm{H}_{2} \mathrm{~S}\right)$ and bacterial activity on the surface of concrete pipes, resulting in the failure of sewer infrastructure. Since the atmospheric conditions inside confined sewers are not sensor friendly, there is a need for an adaptation of current sensor technologies or development of new innovative sensor technologies. For the reason that highly toxic components in sewer gas induces Occupational Health and Safety (OHS) issues, a custom-built smart sensing framework with capabilities of deploying an intelligent autonomous robot can be significantly beneficial in tracking the rate of corrosion.
Several models have been developed previously to predict the possible failure of sewer pipes due to $\mathrm{H}_{2} \mathrm{~S}$ influenced corrosion. However, those models lack the reliable data of key parameters [1]. It has been identified that one of the main factors that govern the high rate of $\mathrm{H}_{2} \mathrm{~S}$ corrosion is moisture content on the walls of sewer pipes [2][3]. In context to aforementioned factor, the objective is to devise a sensor system with reliable sensing performance for measuring the moisture of concrete on the surface of interest. Those sensor data will provide salient information to the existing models of predictive analytics for sewer corrosion.

Current research has reported the relative humidity measurements of the sewer atmosphere by deploying the sensors that are commercially available in the market [4]. However to date, no sensor system has been reported which is capable of measuring moisture on the sewer walls inside confined sewers. As the sewer concrete corrosion incur losses that are estimated to be in the order of billions of dollars per year [5], the wastewater governing bodies are seeking an applicationspecific sensor framework that performs the measurements of interest in the given ambiance.

The work reported in this paper focuses on presenting an analytical model and data-driven approach using Gaussian Process (GP) modeling for predicting the concrete moisture. In this study, the experimental measurements of the electrical resistivity were used to determine the moisture conditions of a concrete. The experimental measurements data were used to train the GP model for high-quality prediction. The data from the analytical model and prediction model was also studied. The work described in this paper is a furtherance of work carried out by [6][7], where the behavior of $\mathrm{pH}$, resistance and moisture at various depths was studied.

The remainder of the paper is organized as follows. Section 2 provides the theoretical background. Section 3 elucidates the analytical model. Section 4 illustrates the experimental data collection methods. Section 5 explains the interpretation of data. Section 6 formulates the GP model. Section 7 presents the results with analyses, followed by conclusions in Section 8 . 


\section{Theoretical Background}

A recent research study has established the reliance on the rate of corrosion ( $\mathrm{mm}$ year $^{-1}$ ) as a functional relationship with the concentration of $\mathrm{H}_{2} \mathrm{~S}$ (ppm), relative humidity $(\%)$ and temperature (Kelvin) in the sewer atmosphere [8]. The bacterium that dwells on the sewer concrete surface oxidizes the gas phase $\mathrm{H}_{2} \mathrm{~S}$ into aqueous sulphuric acid $\left(\mathrm{H}_{2} \mathrm{SO}_{4}\right)$, which in turn reacts with the cement component of concrete [9]. The aqueous by-product that was produced as a result of the bacterial activity is responsible for filling the capillary pores of the sewer concrete. As the bacterial activity increases, the contained liquid within the pores of concrete penetrates deeper and eventually deteriorates embedded rebar. Since the production of aqueous by-product occurs on the surface of the sewer concrete, it is vital to measure the surface properties such as temperature and moisture of the sewer concrete. The measured data can be used to fit into the corrosion prediction model proposed by [8] as an alternative to relative humidity and gas-phase temperature data.

The changes in the moisture content of a concrete can be monitored by measuring changes in the electrical resistance of the concrete [10]. The electrical resistivity, which is a reciprocal of electrical conductivity, is an intrinsic property of a concrete. In [11], it was established that the electrical conduction of a concrete is dependent on both porosity of the concrete and the pore solution conductivity. Also, the electrical properties of the concrete are concurrently affected by the environmental parameters such as temperature variation [12] and moisture variation [13][14]. A new concrete health monitoring system was developed by [15] was used to perform in-situ measurements for evaluating the durability of concretes by measuring the electrical conductivity of concrete, an electrical conductivity of pore solution and temperature variations to calibrate electrical sensors. The study in [16] examined the electrical surface resistivity and bulk electrical resistivity for various concrete mixtures and established a correlation between bulk and surface resistivity of concrete.

Archie's Law described in [17] was developed for a sedimentary rock, which correlates the electrical conductivity to its porosity and brines solution. The Archie's law model that includes properties such as electrical resistivity, porosity and tortuosity of the pore network is used in [18] to estimate the appropriate composition of a concrete mixture for attaining recommended electrical resistivity for guaranteeing the durability of the structure, which is exposed to aggressive environments. By using Archie's Law, a correlation between electrical resistivity and volumetric moisture content of municipal solid waste was expressed in [19].

\section{Formulation of an Analytical Model}

In [17], the classical Archie's Law correlates the insitu electrical conductivity, which is the inverse of electrical resistivity of a rock to its porosity and brines solution resistance. Archie's Law is defined in equation (1) shown below.

$$
R_{t}=a \varnothing^{-m} S_{w}^{-n} R_{w}
$$

where $R_{t}$ is the liquid saturated rock resistivity, $R_{w}$ is the brine resistivity, a is the tortuosity factor, $m$ is the cementation exponent of a rock, $n$ is the saturation exponent and $S_{w}$ is the brine saturation.

The research in [18] has proposed a relationship between the total porosity of the concrete material with the electrical resistivity of the concrete by again using the classical Archie's Law, which is shown in equation (2).

$$
R_{t}=a R_{w} \varepsilon^{-m}
$$

where $\varepsilon$ is the total porosity of the concrete material and the exponential in the equations was taken as the other aspects of the microstructure. In [18], $R_{w}$ was taken as the pore solution resistivity. From the literature, the volumetric water content $\theta_{V}$ relationship with the porosity, which was expressed as in equation (3)

$$
\theta_{V}=\varepsilon R_{S}
$$

where $R_{S}$ is the water saturation ratio defining the ratio of the volume of water to the volume of pore space. The literature [19] shows the relationship of volumetric moisture content and electrical resistivity of the solid waste by rewriting the Archie's Law as shown in equation (4).

$$
R_{t}=a R_{w} \theta_{V}^{-m}
$$

The relationship of $R_{t}$ and $\theta_{V}$ was adopted from [19] in this study for the concrete material to formulate an analytical model. Rewriting the above equation (4) for mathematical simplification in the following equations:

$$
\begin{gathered}
\theta_{V}^{-m}=\left[\left(R_{t}\right) /\left(a \cdot R_{w}\right)\right] \\
\theta_{V}=\left[\left(\mathrm{R}_{\mathrm{t}}\right) /\left(\text { a. } \mathrm{R}_{\mathrm{w}}\right)\right]^{[-(1 / m)]} \\
\theta_{V}=\left[\left(\text { a. } R_{w}\right) /\left(R_{t}\right)\right]^{[(1 / m)]}
\end{gathered}
$$

From [18], the value of tortuosity factor $a$ was approximately equal to 1 . 


\section{Experimental Data Collection Methods}

The experimental preparation of concrete samples and methods for measuring environmental variables that were used in this work for interpreting moisture conditions of the concrete are described in the following subsections. All the experiments were carried out in laboratory conditions at CAS Robotics Research Lab, UTS.

\subsection{Preparation of Concrete Samples}

Five concrete samples were used for this experimental program. The dimensions of each concrete sample were $20 \mathrm{~cm}$ (length) x $10 \mathrm{~cm}$ (height) x $5 \mathrm{~cm}$ (width). All the concrete blocks were dried using a microwave oven and left to cool for a few hours to ambient temperature. Two holes were drilled on each concrete sample for placing the electrodes to facilitate electrical resistivity measurements. Before placing the electrodes in the drilled holes of the concrete samples, the dry weight of each concrete sample was recorded. As shown in Figure 1, each concrete sample was placed in a plastic container that was filled with a liquid to a level such that the base of the concrete sample is in contact with the liquid. The liquid level of each container was monitored frequently and kept adding the necessary amount of liquid throughout the experimental program.

The electrodes were placed at a depth of $4.5 \mathrm{~cm}$ from the top surface of the concrete, i.e., $0.5 \mathrm{~cm}$ height from the surface that was exposed to the liquid. The electrodes that were used in the experiment were tampered resistance with dimensions of $75 \mathrm{~mm}$ (Height) x $4 \mathrm{~mm}$ (Width). These electrodes were made up of carbon steel and plated with zinc for corrosion protection. Two electrodes were drilled onto each concrete block with the spacing of $1 \mathrm{~cm}$ apart and each electrode weighed approximately 7.55 grams.

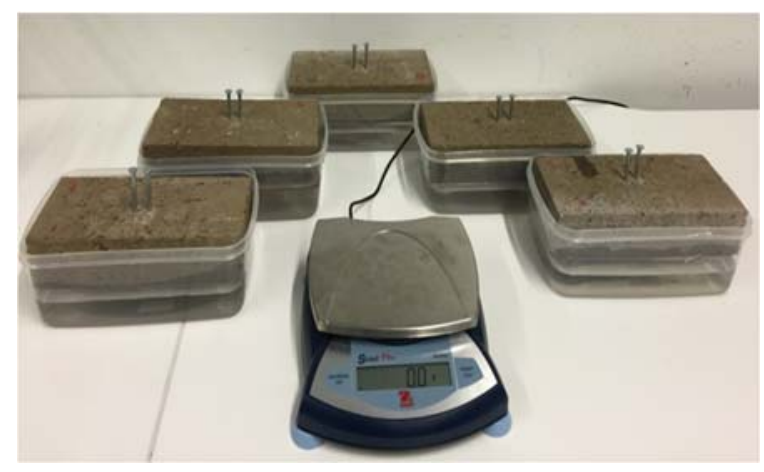

Figure 1. Experimental setup during wetting process

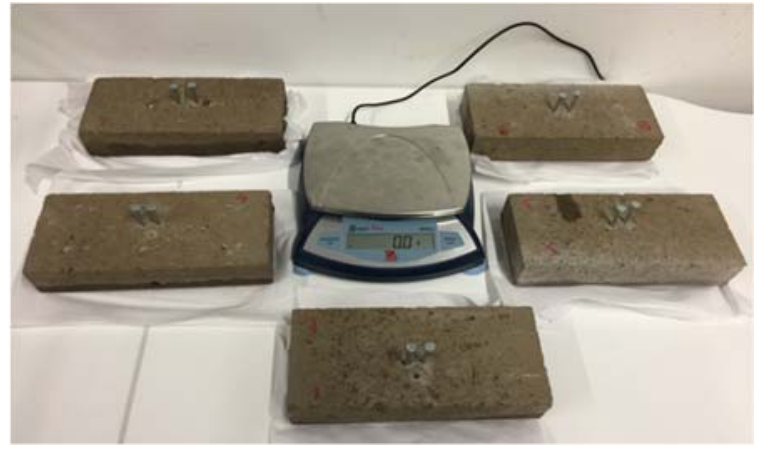

Figure 2. Experimental setup during drying process

\subsection{Approach to Experimental Measurements}

In this subsection, the experimental approach for measuring the variables such as moisture content and electrical resistivity of the concrete samples are described in detail. The measurements of all the variables were taken simultaneously.

The moisture content of the concrete sample was measured by means of gravimetric analysis. Two different methods were practiced to determine the moisture content of concrete samples. The first method to determine the concrete moisture content was through the wetting process. In this method, each concrete sample surface was exposed to a container containing water. As the water ingresses into the concrete from the exposed surface, there is an increase in the mass of the concrete sample. Then, the concrete sample was taken out of the container with care and its surface was wiped gently with paper towel to remove excess water. Subsequently, the mass of the concrete sample was recorded by using a weighing scale (OHAUS SP-6001) that has a precision of 0.1 grams. The second methodology used in the experimental program for determining moisture content was through the drying process. In this method, the concrete sample was removed from the container and allowed to dry in laboratory conditions. While drying, the mass of wet concrete sample decreases and its decreasing mass were recorded. The value of $m_{w}$ increases during the wetting process and it decreases while drying. By using the gravimetric method, the determination of moisture content $\theta_{\mathrm{G}}$ of the concrete sample for wetting and drying process can be defined as in equation (8):

$$
\theta_{G}=\frac{m_{w}-m_{d}}{m_{d}} \times 100
$$

where $\theta_{\mathrm{G}}$ is the gravimetric moisture content, $m_{w}$ is the mass of the concrete sample in wet condition and $m_{d}$ is the mass of the concrete in dry condition. 
An electrical resistivity test was conducted on all the concrete samples. As covered in section 4.1, a pair of electrodes was used to facilitate electrical resistivity measurements while recording moisture content measurements during wetting and drying process. The electrodes were inserted in a way such that the tips of the electrodes were in contact with the concrete depth of $0.5 \mathrm{~cm}$ height from the surface that was exposed to water.

\section{Interpretation of Experimental Data}

This section explains the interpretation and analysis of data that were obtained during the experimental program. The moisture content measurements of the concrete samples that were obtained through the wetting and drying process by using gravimetric method were used to determine the volumetric moisture content. As in [20], the volumetric moisture content $\theta_{V}$ of the material can be determined by using the equation (9):

$$
\theta_{V}=\frac{\rho_{d}}{\rho_{w}} \times \theta_{G}
$$

where $\theta_{\mathrm{G}}$ is the gravimetric based moisture content, $\rho_{w}$ is the density of the liquid and $\rho_{d}$ is the density of concrete sample in a dry condition, which is determined using the formula in equation (10):

$$
\rho_{d}=\frac{m_{d}}{\text { volume }} \quad\left(\mathrm{g} / \mathrm{cm}^{3}\right)
$$

The volume of each concrete sample was $1000 \mathrm{~cm}^{3}$ $(20 \mathrm{~cm} \times 10 \mathrm{~cm} \times 5 \mathrm{~cm})$. Since the electrodes were drilled and placed at a height of $0.5 \mathrm{~cm}$ from the concrete surface that was exposed to aqueous solution, the height of the concrete $5 \mathrm{~cm}$ is reduced to $0.5 \mathrm{~cm}$ and then the new volume of the concrete was calculated to be $200 \mathrm{~cm}^{3}$ and it was used to calculate the $\rho_{d}$ values that are presented in Table 1. The liquid used for the experiment was the water and the value of $\rho_{w}$ was taken to be $1.0 \mathrm{~g} / \mathrm{cm}^{3}$.

Table 1 Dry Density of Concrete Samples

\begin{tabular}{cc}
\hline Concrete Number & Dry Density $\left(\rho_{d}\right)$ \\
\hline 1 & $21.961 \mathrm{~g} / \mathrm{cm}^{3}$ \\
2 & $21.567 \mathrm{~g} / \mathrm{cm}^{3}$ \\
3 & $22.121 \mathrm{~g} / \mathrm{cm}^{3}$ \\
4 & $21.808 \mathrm{~g} / \mathrm{cm}^{3}$ \\
5 & $21.648 \mathrm{~g} / \mathrm{cm}^{3}$ \\
\hline
\end{tabular}

The determined data of volumetric moisture content $\theta_{V}$ of concrete samples from $\theta_{\mathrm{G}}$ were interpreted with the data of electrical resistivity measurements of concrete samples during the wetting and drying process of moisture measurements. The concrete samples that were numbered from 1 to 4 were used for training, while the data-driven model and concrete sample numbered 5 was used to test the prediction model. Figure 3 and Figure 4 presents the volumetric moisture profile during wetting and drying process and the interpreted electrical resistivity data.

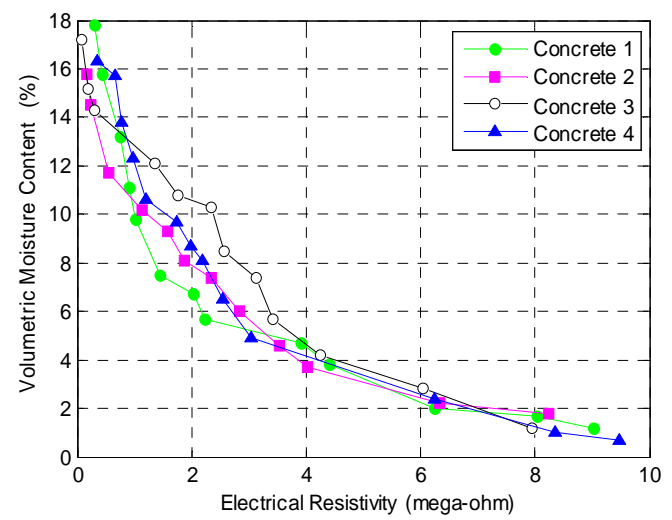

Figure 3. The plot of electrical resistivity data versus volumetric moisture content profile during the wetting process.

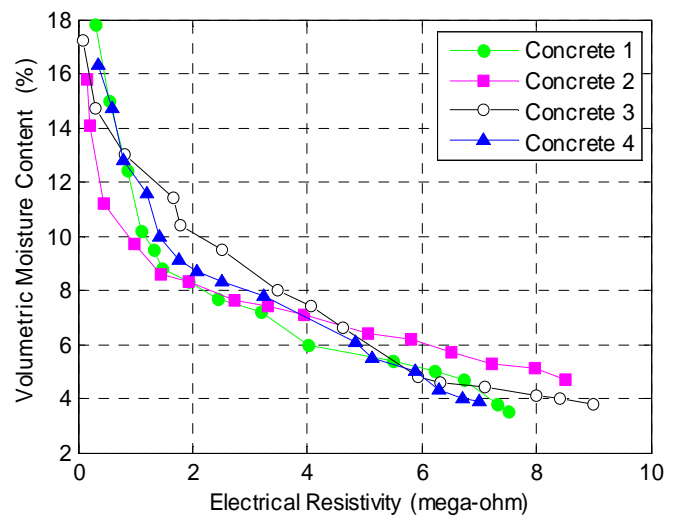

Figure 4. The plot of electrical resistivity data versus volumetric moisture content profile during the drying process.

It can be observed from the interpreted experimental data of electrical resistivity and volumetric moisture content shown in Figure 3 and Figure 4 that there is a decrease in electrical resistivity measurements and increases in volumetric moisture content, during both wetting and drying process and it is evident that the trend was similar for all the concrete samples. 


\section{Data-driven Approach Using Gaussian Process Regression (GPR) Modeling}

This section describes the data-driven machine learning approach using Gaussian Process Regression (GPR) for predicting the moisture conditions on the concrete. The Gaussian Process (GP) modeling approach in [21] was used in this study to train the nonparametric model that obtains electrical resistivity $(R)$ values as inputs and predicts the percentage volumetric moisture content $(V M C)$. The motive is to learn the function $f$ in the form as in equation (11)

$$
V M C=f(R)+\xi
$$

where $\xi$ is the uncertainty. Let $X_{1}, X_{2}, X_{3}$ and $X_{4}$ be the electrical resistivity data and $Y_{1}, Y_{2}, Y_{3}$ and $Y_{4}$ be the volumetric moisture content of four different concrete samples from the experimental program during wetting and drying process. The data from each concrete was combined together to increase the training data points for GP modeling. Now, $X=\left[X_{1}+X_{2},+X_{3}+X_{4}\right]$ and $Y=\left[Y_{1}+Y_{2},+Y_{3}+Y_{4}\right]$.

Let $[X, Y]$ be the training data set input to GP model, where $X=\left[x_{1}, x_{2}, x_{3} \ldots x_{m}\right]^{\mathrm{T}}$ and $Y=\left[y_{1}, y_{2}, y_{3} \ldots y_{m}\right]^{\mathrm{T}}$. The $X$ is a matrix of training input extracted from electrical resistivity measurements having $x_{i}=\left[R_{i}\right]^{T}$ and $i(1 \leq i \leq \mathrm{m})$, where is $i$ is an integer and $m$ is number of captured data points. The $Y$ is the training vector label data of volumetric moisture content corresponding to the training targets having $y_{i}=V M C_{i}$. Likewise, $\left[X^{*}, Y^{*}\right]$ be the testing data set to the GP model, where $X^{*}=\left[x^{*}\right.$, $\left.x_{2}, x_{3} *_{3} \ldots x_{n}\right]^{\mathrm{T}}$ and $Y^{*}=\left[\mathrm{y} *_{1}, y^{*}, y^{*}{ }_{3} \ldots y^{*}\right]^{\mathrm{T}}$. The $X^{*}$ is a matrix containing testing data and $Y^{*}$ is a vector containing predicted outputs corresponding to $X^{*}$. Once the function has been learned using $[X, Y]$, the $f$ can be used to predict $Y^{*}$ for testing data $X^{*}$ as in equation (12)

$$
V M C^{*}=f\left(R^{*}\right)+\xi^{*}
$$

From [21], a kernel $K(X, X)$ having elements $k_{i, j}=k\left(x_{i}, x_{j}\right)$ was selected to apply GP modeling technique for the non-linear regression problem in this study. After assessing number of generally used kernels, a squared-exponential kernel having a one-dimensional form with free parameters as in (13) was chosen for this work.

$$
k\left(x_{i}, x_{j}\right)=\alpha^{2} \exp \left\{-\frac{1}{2 \beta^{2}}\left(x_{i}-x_{j}\right)^{2}\right\}
$$

where the $\alpha$ and $\beta$ in (13) are free parameters that were learned from the training input data set $[X, Y]$. Normally, the free parameters are the hyper-parameters. The technique of training GPR model in [21] by minimizing the negative log marginal likelihood in (14) with respect to the hyperparameters $\theta=\left\{\alpha, \beta, \sigma_{n}\right\}$ was performed while formulating the prediction model.

$$
-\log p(Y \mid X, \theta)=\frac{1}{2} Y^{T} \Sigma^{-1} Y+\frac{1}{2} \log \left|\sum\right|+\frac{m}{2} \log (2 \pi),
$$

The covariance function denoted by $\sum$ in (14) is expressed in (15)

$$
\sum=K(X, X)+\sigma_{n}^{2} I
$$

The basic GPR model equations are given by (16) and (17).

$$
\begin{aligned}
\mu^{*}= & K\left(X^{*}, X\right)\left\{K(X, X)+\sigma^{2}{ }_{n}\right\}^{-1} y \\
\Sigma^{*}= & K\left(X^{*}, X^{*}\right)+\sigma^{2} I-K\left(X^{*}, X\right) \\
& \left\{K(X, X)+\sigma^{2}{ }_{n} I\right\}^{-1} K\left(X, X^{*}\right)
\end{aligned}
$$

The predicted volumetric moisture content $\left(Y^{*}\right)$ for the testing input vector $\left(X^{*}\right)$ will be therefore given by the mean of the posterior distribution $\mu^{*}$ and the associated uncertainty will be given by the covariance $\sum^{*}$. The functional block diagram in Figure 5 describes the formulation of GPR model for the data-driven approach.

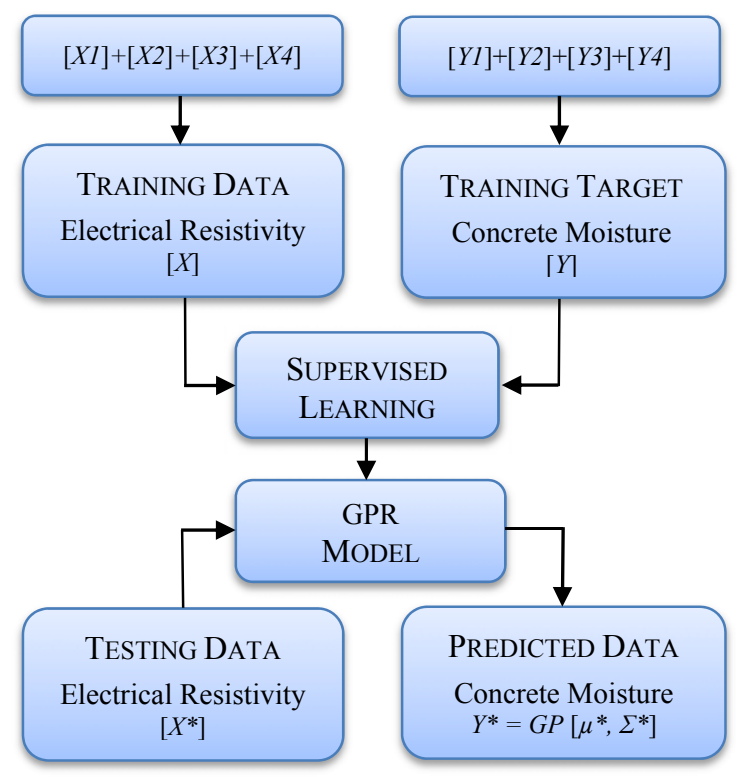

Figure 5. Functional Block Diagram: Training GPR Model and Predicting Output. 


\section{$7 \quad$ Results and Analysis}

This section presents the results of data-driven prediction modeling using GPR and analysis of the formulated analytical model. The experimental data from the wetting and drying phase of concrete were combined together to increase the number of training data input set feature for GP modeling. Figure 6 shows the obtained input training data in 2D for GP modeling.

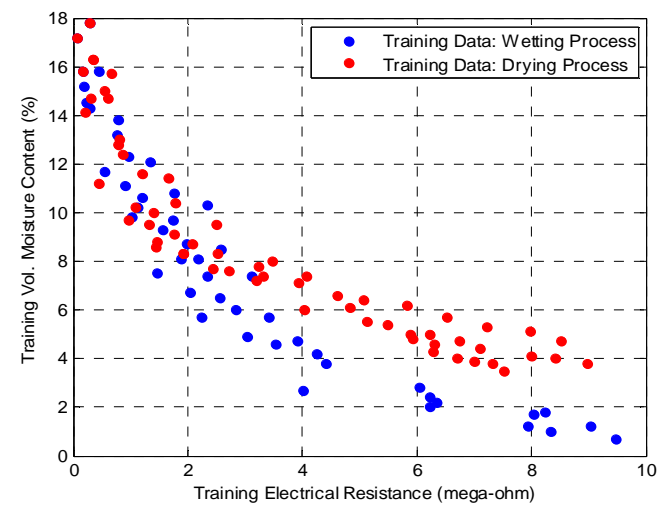

Figure 6. Learned GP model with training data.

For the given electrical resistivity testing data from wetting and drying process, the volumetric moisture content was predicted. Figure 7 shows the Gaussian Process model predictions in 2D.

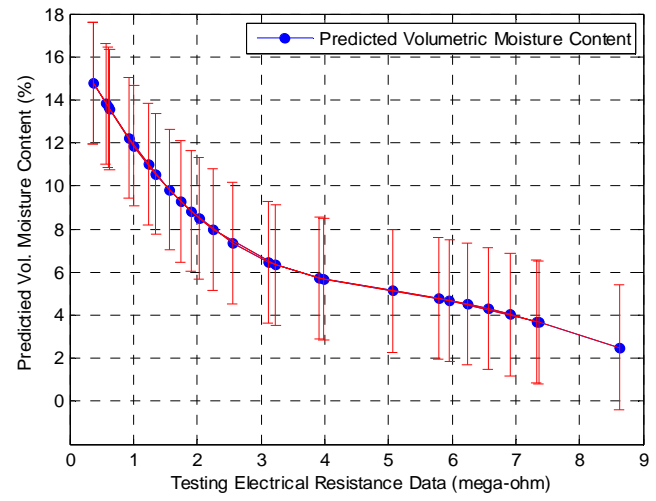

Figure 7. Gaussian Process predictions in 2D

The behavior of predicted volumetric moisture content with respect to training volumetric moisture content data is shown in Figure 8. It can be observed that the Gaussian Process predicted data and the training data fits within the $2 \sigma$ uncertainty bounds as it learns the function $f$ from equation (12).

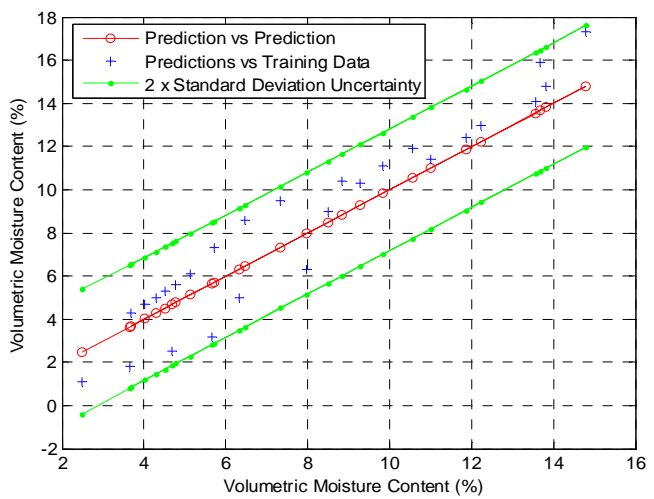

Figure 8. The behavior of predicted values corresponding to training data.

The statistical metric, Root-Mean-Square (RMS) error metric was used as a criterion to compute prediction performance. The RMS error between the GP predicted data and measured data (testing data) is $1.5020 \%$ while the mean absolute error is $1.3412 \%$. Figure 9 compares predictions to measured values (testing data) from the concrete that was used for testing purpose.

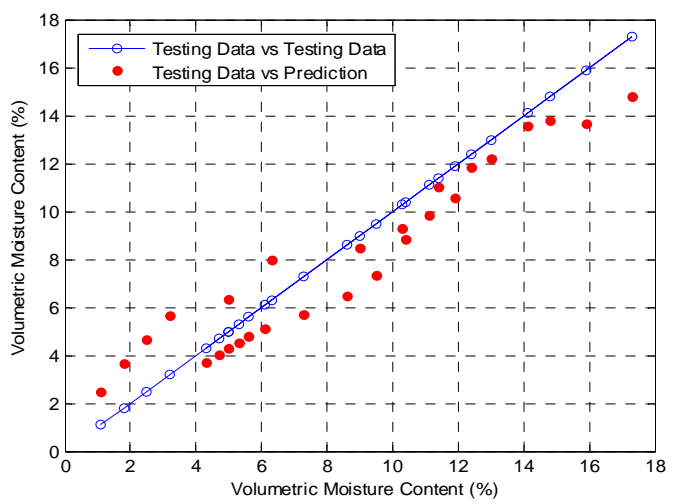

Figure 9. The behavior of Predictions vs. Measured values (testing data).

The analytical model curve in Figure 10 is based on the measurements of values that were substituted in the analytical model equation (7). The aqueous solution that was used to soak the concretes has a resistance value that was measured to be $R_{w}=35 \mathrm{KOhm}$. As from the literature, the tortuosity factor $(a)$ was chosen as 1 and the cementation exponent was adjusted to be 1.65 in this study. The cementation exponent $m$ for rock varies from 1.3 to 2.2 [22]. By choosing arbitrary values of resistivity from $0.5 \mathrm{MOhm}$ to $10 \mathrm{MOhm}$, the volumetric 
moisture content of concrete material was determined using analytical model equation (7). Figure 12 presents the plot of analytical model data with experimental data that was obtained during the wetting-drying process, and Figure 11 presents the curve of predicted moisture profiles and determined curve from the analytical model equation.

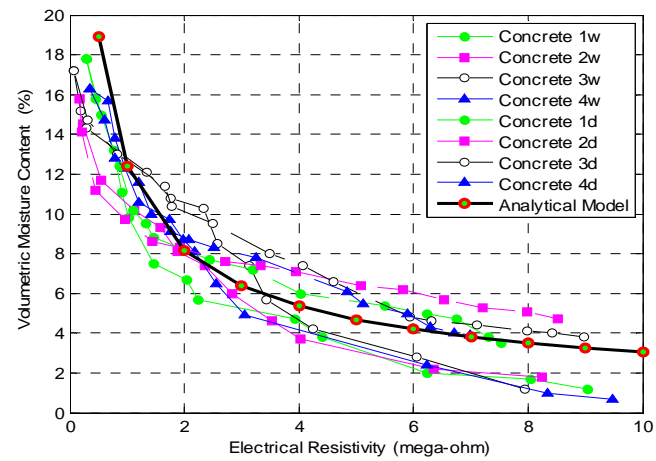

Figure 10. The plot of analytical model data with the experimental measurement data.

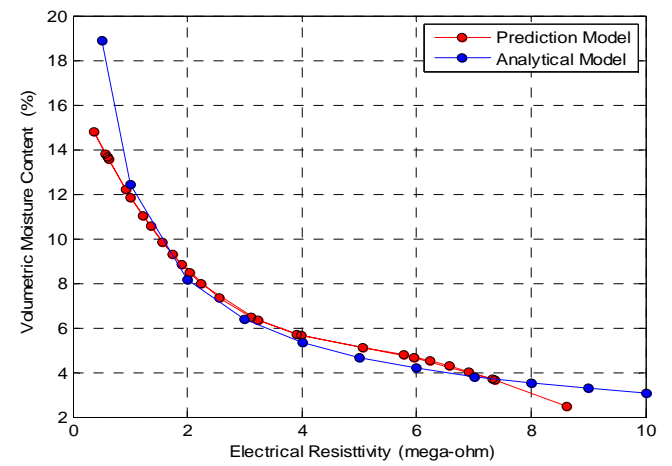

Figure 11. The plot of predicted model data and analytical model data.

From Figure 7, it can be observed from the predicted data has a high value of error bounds. This is primarily because of the experiment that was carried out on various concrete samples, which may differ in porosity, concrete material composition and water-cement ratio. The key element of the research work was to estimate the levels of moisture conditions $(0 \%, 25 \%, 50,75$ and $100 \%$ ). Having the high value of error bound in predicted data may not significantly affect accuracy in predicting moisture levels. As mentioned in the previous section, the majority of concrete corrosion happens on the crown of sewer concrete pipe. As we intend to mount the sensory unit in the crown of the sewer pipe, we may need corrosion resistant bolts to mount sensing enclosure unit. These bolts may be also used as electrodes to obtain the moisture conditions of the concrete. The resistivity based measurements need calibration, as it is susceptible to errors due to other chemicals that are present in sewer walls.

\section{Conclusion and Future Work}

The study in this paper presented a data-driven machine learning approach for predicting the volumetric moisture conditions of concrete samples based on the interpreted electrical resistivity measurements data that were obtained by embedding the electrodes near to the concrete surface, which was exposed to the aqueous solution. Also, the study investigated the use of electrical resistivity measurements for determining the moisture conditions of concrete samples. The results of experimental data show that the electrical resistivity of concretes increases with the decrease in volumetric moisture content data during both wetting and drying process. The GPR modeling by training data from both wetting-drying processes measurements was presented, analyzed and their prediction performance was assessed via RMS error. The data obtained from the analytical model was compared and analyzed with the data-driven predicted data and experimental data. The experimental methodology carried out in this paper provides the preliminary results on moisture content profile on concretes during wetting and drying process. Further study needs to be conducted for evaluating the reliability of resistance based measurements on varying surface $\mathrm{pH}$ and ambient $\mathrm{H}_{2} \mathrm{~S}$ levels.

\section{Acknowledgement}

This publication is an outcome from the Data Analytics on Sewers Project funded by Sydney Water Corporation, Melbourne Water Corporation, Water Corporation (WA) and South Australian Water Corporation. The research participants are NICTA (lead), University of Technology Sydney and The University of Newcastle. Karthick Thiyagarajan receives UTS President's Scholarship (UTSP) and International Research Scholarship (IRS) from The University of Technology Sydney.

\section{References}

[1] Romanova A., Mahmoodian M. and Alani M.A. Influence and interaction of temperature, H2S and $\mathrm{pH}$ on concrete sewer pipe corrosion. International Journal of Civil, Architectural, Structural, Urban Science and Engineering, 8(6): p. 592-595, 2014. 
[2] Mori T., Nonaka T., Tazaki K., Koga M., Hikosaka Y. and Noda S. Interactions of nutrients, moisture and $\mathrm{pH}$ on microbial corrosion of concrete sewer pipes. Water Research, 26(1): p. 29-37, 1992.

[3] Joseph A.P., Keller J. Bustamante H. and Bond P.L. Surface neutralization and H2S oxidation at early stages of sewer corrosion: Influence of temperature, relative humidity and $\mathrm{H} 2 \mathrm{~S}$ concentration. Water Research, 46(13): p. 42354245, 2012.

[4] Wells P. and Melchers R.E. Microbial Corrosion of Sewer Pipe in Australia-Initial Field Results. In Proceedings of the 18th International Corrosion Congress, November 2011.

[5] Jiang G., Wightman E., Donose B.C., Yuan Z., Bond P.L. and Keller J. The role of iron in sulfide induced corrosion of sewer concrete. Water research, 49: p. 166-174, 2014.

[6] Thiyagarajan K. and Kodagoda S. SMART monitoring of surface temperature and moisture content using multisensory data fusion. In Proceedings of the Cybernetics and Intelligent Systems (CIS) and IEEE Conference on Robotics, Automation and Mechatronics (RAM), 2015 IEEE 7th International Conference on, pages 222-227, 2015.

[7] Thiyagarajan K., Kodagoda S. and Ulapane N. Data-driven Machine Learning Approach for Predicting Volumetric Moisture Content of Concrete Using Resistance Sensor Measurements. In Proceedings of the Industrial Electronics and Applications (ICIEA), 2016 IEEE 11th Conference on, pages 1288-1293, 2016.

[8] Wells T. and Melchers R. Modelling concrete deterioration in sewers using theory and field observations. Cement and Concrete Research, 77: p. 82-96, 2015.

[9] Zhang L., Schryver P.D., Gusseme B.D., Muynck W.D., Boon N. and Verstraete W. Chemical and biological technologies for hydrogen sulfide emission control in sewer systems: a review. Water research, 42(1): p. 1-12, 2008.

[10] Parrott L. A review of methods to determine the moisture conditions in concrete. 1990.

[11] Xiao L. and Li Z. Early-age hydration of fresh concrete monitored by non-contact electrical resistivity measurement. Cement and Concrete Research, 38(3): p. 312-319, 2008.

[12] Whittington H.W., McCarter J. and Forde M.C. The conduction of electricity through concrete. Magazine of concrete research, 33(114): p. 48-60, 1981.

[13] McCarter W., Ezirim H. and Emerson M. Properties of concrete in the cover zone: water penetration, sorptivity and ionic ingress. Magazine of Concrete Research, 48(176): p. 149-156, 1996.

[14] Rajabipour F., Weiss J., Shane J.D., Mason T. and Shah S.P. Procedure to interpret electrical conductivity measurements in cover concrete during rewetting. Journal of Materials in Civil Engineering, 17(5): p. 586-594, 2005.

[15] Rajabipour F., Sant G. and Weiss J. Development of electrical conductivity-based sensors for health monitoring of concrete materials. In Proceedings of the TRB Annual Conference, 2007.

[16] Ghosh P. and Tran Q. Correlation Between Bulk and Surface Resistivity of Concrete. International Journal of Concrete Structures and Materials, 9(1): p. 119-132, 2015.

[17] Archie G.E.The electrical resistivity $\log$ as an aid in determining some reservoir characteristics. Transactions of the AIME, 146(01): p. 54-62, 1942.

[18] Andrade C. and D'Andréa R. Concrete mixture design based on electrical resistivity. In Proceedings of the Second International Conference on Sustainable Construction Materials and Technologies. 2010.

[19] Grellier S., Reddy K.R., Gangathulasi J., Adib R. and Peters C. Correlation between electrical resistivity and moisture content of municipal solid waste in bioreactor landfill. Geotechnical Special Publication, 163: p. 1-14, 2007.

[20] Alam M.N., Bhuiyan R.H., Dougal R. and Ali M. Concrete moisture content measurement using interdigitated near-field sensors. Sensors Journal, IEEE, vol. 10, pp. 1243-1248, 2010.

[21] Rasmussen C.E. Gaussian processes for machine learning. 2006.

[22] Breysse D. Non-Destructive Assessment of Concrete Structures: Reliability and limits of Single and Combines Techniques. Springer, 2012. 